\title{
The c-Fes protein tyrosine kinase as a potential anti-angiogenic target in cancer
}

\author{
Shigeru Kanda ${ }^{1,3}$ and Yasuyoshi Miyata ${ }^{2}$ \\ ${ }^{1}$ Department of Molecular Microbiology and Immunology, Division of Endothelial \\ Cell Biology, and ${ }^{2}$ Department of Nephro-urology, Nagasaki University Graduate \\ School of Biomedical Sciences, 1-7-1 Sakamoto, Nagasaki 852-8501; \\ ${ }^{3}$ Department of Experimental and Clinical Laboratory Medicine, National \\ Hospital Organization, Nagasaki Hospital, 41-6 Sakuragi-machi, Nagasaki \\ 851-0251, Japan
}

Running title: Roles of c-Fes in angiogenesis

Keywords: c-Fes, endothelial cell, FGF, angiopoietin, SDF-1alpha, IL-8, VEGF

All corresponding to:

Shigeru Kanda, M.D., Ph.D.

National Hospital Organization, Nagasaki Hospital, 41-6 Sakuragi-machi,

Nagasaki 851-0251, Japan.

Phone: +8195823 2261

Fax: +8195828 2616

E-mail: skanda-jua@umin.net 


\section{TABLE OF CONTENTS}

1. Abstract

2. Introduction

2.1. Angiogenic cellular processes by endothelial cells

2.2. VEGF-A as a central player in angiogenesis

2.3. Resistance to therapy targeting VEGF-mediated signaling

3. Roles of c-Fes in angiogenic processes induced by a variety of proangiogenic factors

3.1. FGF

3.2. Angiopoietins

3.3. SDF-1alpha

3.4. IL-8

3.5. VEGF

4. Future directions for therapeutic applications

5. Acknowledgments

6. References 


\section{ABSTRACT}

Angiogenesis is implicated in many pathological conditions, including cancer progression. Novel approaches have enabled an understanding of how endothelial cellular processes are regulated in vivo during angiogenesis. Key players in angiogenesis are vascular endothelial growth factors (VEGFs) and Notch ligands. However, mechanisms of angiogenic responses by other proangiogenic factors in vivo are largely unknown. Research using cultured endothelial cells has shown that c-Fes is involved in the activation of phosphoinositide 3-kinase (PI3-kinase) downstream of a variety of cytokine receptors. The PI3-kinase/c-Akt pathway regulates cell survival, migration, and morphological differentiation of endothelial cells during angiogenesis, and c-Fes thus may be a potential target of anti-cancer therapy, especially for patients with anti-VEGF refractory cancer. In addition, a number of experiments have shown that a bone marrow-derived monocytic lineage regulates angiogenesis, and c-Fes is also expressed in these cells. Roles for c-Fes during angiogenesis will be the focus of extensive research in the future. 


\section{INTRODUCTION}

\subsection{Angiogenic cellular processes by endothelial cells}

Angiogenesis, or new blood vessel formation from pre-existing blood vessels, is a prerequisite for many normophysiological and pathophysiological conditions, such as cycles in female reproductive organs, embryonic development, regenerative medicine, collateral formation after occlusion of arteries, age-related macular degeneration, proliferative retinopathies, and malignant tumor growth/progression (1,2). Although angiogenesis is favorable in implantation of engineered tissues for regenerative medicine and in collateral formation as support for obstructive arteries, inhibition of angiogenesis leads to disease control for some types of eye diseases and advanced malignant tumors.

Classically, angiogenesis has been described as resulting from a series of cellular processes involving vascular endothelial cells, including the production of proteases to digest the basement membrane of blood vessels, thus allowing endothelial cells to migrate into surrounding tissue, survive, proliferate, and form a lumen (3). Cultured endothelial cells can be used to examine this process separately, and in this context, fibroblast growth factors (FGFs), vascular endothelial growth factors (VEGFs), hepatocyte growth factor (HGF), and angiopoietins (Ang) have been found to stimulate almost all angiogenic cellular processes (4-7). In addition, the biochemical relevance of intracellular signaling molecules in each process has also been identified, such as phosphoinositide 3-kinase (PI3-kinase) and its downstream kinase, c-Akt, extracellular signal-regulated kinases/mitogen-activated protein kinase (ERK/MAPK), the c-Src family kinases, and c-Fes (8-11).

\subsection{VEGF-A as a central player in angiogenesis}

The VEGF family consists of five members, VEGF-A, -B, -C, -D, and placental growth factor (PIGF), and their signals are transduced into cells via VEGF receptor (VEGFR)-1, -2, and -3 $(12,13)$. The prototype VEGF, VEGF-A, binds to both VEGFR-1 and -2, whereas VEGF-B and PIGF exclusively bind to VEGFR-1. VEGFR-3 has been identified as a lymphatic endothelial cell receptor for VEGF-C and -D, which also bind to VEGFR-2. In general, the functional receptor for VEGF-A is VEGFR-2, while VEGFR-1 is thought to be a decoy 
receptor to regulate the concentration of VEGFR-2-associated VEGF-A (14).

Recently, excellent technical progress has enabled examination of the angiogenic cellular processes of endothelial cells in vivo. In an early postnatal retinal model, tip cells, endothelial cells migrating in the leading edge of sprouts, and stalk cells, endothelial cells following tip cells, behaved distinctly in response to VEGF treatment (15). Tip cells migrate using filopodia, resulting in branching and network formation of capillaries, and their migration depends on a gradient of VEGF-A. By contrast, sprout stalk cells respond with proliferation depending on the VEGF-A concentration. These processes are regulated through interaction between VEGF-A and VEGFR-2 (15). Delta-like 4 (DII4), signaling through its receptor Notch1, inhibits VEGFR-2-mediated tip cell formation, whereas its inhibition maintains the stalk cell phenotype $(15,16)$. Dll4/Notch1 signaling induces expression of VEGFR-1, but it inhibits VEGFR-2 expression $(17,18)$. Another Notch1 ligand, Jagged1 (Jag1), has the opposite effect on Notch1-inhibited tip cell formation (19). Jag1 antagonizes DIl4-mediated Notch1 signals, which are regulated through modification of Notch1 by glycosyltransferases from the Fringe family. Jag1 is expressed in stalk cells, promoting their activation and maintaining the VEGF response.

Notch plays a role in VEGFR-3 regulation, as well. In the microvessels of early postnatal retina and growing tumors, VEGFR-3 becomes unregulated with inhibition of Notch signaling $(20,21)$. Blockade of VEGFR-3 inhibits branching angiogenic sprouts (21), suggesting that VEGFR-3 is also required for pathophysiological angiogenesis. Expression of VEGFR-3 decreases when Jag1 is deleted in tip cells (19).

Ephrin-B2, a ligand of EphB4 receptor tyrosine kinase, also plays a role in this pathway and has been shown to regulate the internalization of VEGFR-2 and $-3(22,23)$. Signaling via VEGFR-2 and -3 requires their internalization, and the PDZ domain of ephrin-B2 is responsible for this dynamin-dependent internalization (22). However, the molecular mechanisms underlying how the PDZ domain of ephrin-B2 controls this internalization and subsequent alteration of signal transduction pathways via VEGFRs remain unknown. Endothelial cells deficient in ephrin-B2 exhibit reduced chemotaxis toward VEGF-A, but not toward FGF (23), suggesting that signaling via the FGF receptor (FGFR) is distinct from VEGFR. 
Proangiogenic factors in addition to VEGF, such as FGF-2, HGF, Ang, and interleukin (IL)-8, can induce angiogenesis, and a number of tumor cells produce these factors. Nevertheless, their effect on the processes of tip cells and stalk cells remains unknown, as does whether tip and stalk cells are also key players for this proangiogenic factor-driven pathological angiogenesis.

In addition to angiogenesis, vasculogenesis has been found to be involved in tumor growth and progression. Vasculogenesis is defined as novel blood vessel formation without sprouting from preexisting vessels, especially by circulating endothelial precursor cells or endothelial cell progenitors from bone marrow (24). Also, vessel cooption, in which a preexisting vessel serves as a substitute for a novel blood supply (25), may contribute to supply oxygen and nutrients to hypoxic tumor tissue.

\subsection{Resistance to therapy targeting VEGF-mediated signaling}

VEGF receptor-2 (VEGFR-2), the most functional of all of the VEGF receptors, is exclusively expressed in activated endothelial cells. Tumor tissue is usually hypoxic because of the rapid growth of tumor cells, and expression of VEGF-A is regulated through hypoxia-inducible factor-dependent mechanisms. The roles of VEGF-ANEGFR-2 signaling in tumor progression have been studied extensively, and it is now widely recognized that VEGF-A is a key player in tumor angiogenesis. Initially, it was thought that anti-angiogenic therapies does not develop resistance because the targeted endothelium is genetically stable (26). Therefore, many VEGF-targeting therapies, such as blocking antibodies against VEGF-A (e.g., bevacizumab) and small molecular weight synthetic protein kinase inhibitors for VEGF receptors (e.g., sunitinib and sorafenib), have been used in many types of cancers (27). In general, however, the period of effectiveness is limited, and resistance to these therapies through multiple mechanisms has been reported $(28,29)$.

Among these mechanisms of resistance, alteration of VEGF-A-dependent angiogenesis to other proangiogenic factor-dependent angiogenesis is one of the best-described. Many preclinical models and clinical studies have shown that FGF-2, Ang2, stromal cell-derived factor-1alpha (SDF-1alpha), IL-8, platelet-derived growth factor-C (PDGF-C), and endocrine gland-derived VEGF homologue Bv8 are candidate proangiogenic factors in the 
resistance to anti-VEGF-A therapies (30-36). Specific receptors for FGF-2, Ang2, and PDGF-C are protein tyrosine kinases, whereas receptors for SDF-1alpha, IL-8, and Bv8 are seven-transmembrane, G-protein-coupled receptors $(37,38)$. To inhibit anti-VEGF-refractory tumor growth, targeting intracellular signaling molecules/pathways commonly acting downstream of multiple proangiogenic factor receptors would be a favorable strategy for blocking several proangiogenic-dependent signals simultaneously.

The Ras/ERK pathway, PI3-kinase/c-Akt pathway, and a number of cytoplasmic protein tyrosine kinases (PTKs) including c-Src-, C-Jak-, and c-Fes-family members are activated downstream of these transmembrane angiogenic receptors. These intracellular signaling molecules exert critical roles in proliferation, motility, and survival of tumor cells and tumor-associated fibroblasts. Thus, targeting these molecules represents a promising strategy to effectively block angiogenesis and subsequent tumor progression. Expression of the c-Fes RTK is tissue restricted with highest levels in the monocytic hematopoietic and endothelial lineages, as well as some neuronal epithelial cell types (39). In addition, c-Fes is also expressed in several types of tumor cells. It is therefore possible that if c-Fes involvement in angiogenic processes is confirmed using endothelial cells treated with particular proangiogenic factors, c-Fes may become a candidate target for anti-angiogenic therapy and anti-tumor therapy. Recent findings regarding the role of c-Fes in endothelial cells are described in detail below. 


\section{ROLES OF C-FES IN ANGIOGENIC PROCESSES INDUCED BY A VARIETY OF PROANGIOGENIC FACTORS}

\subsection{FGF}

The FGF family is composed of 22 structurally related members (40). Four FGF receptor tyrosine kinases have been identified, designated as FGFR1 to 4 (41). While FGF-2 (basic FGF) lacks a signal peptide (the mechanisms underlying its secretion are still undefined), it has been extensively studied in the field of vascular biology (42). The major receptor for FGF in endothelial cells is FGFR1. In some endothelial cells, FGFR2 is also expressed. FGF-2 has a high affinity for the alternatively spliced IIIC isoform of both FGFR1 and 2 (43). While FGFR1 transduces signals leading to proliferation, protease production, migration, and tubule formation (morphological differentiation) (44), signals via FGFR2 seem to be required only for endothelial cell migration (45). FGFR1 is expressed in a variety of cell types, suggesting that FGF-2 is a pleiotropic factor acting not only on endothelial cells but also on a number of different types of cells. Hypoxia does not generally regulate FGF-2 expression, and targeted deletion of the fgf-2 gene results in no lethal vascular defect in mutant mice (46, 47). In addition, the precise role of FGF-2 in tumor progression and angiogenesis in human cancers remains elusive. Recently, several investigations with preclinical models have shed light on FGF-2, suggesting that it may contribute to the establishment of resistance to VEGF-directed anti-angiogenic therapy (30). Therefore, regardless of its potent proangiogenic activity, FGF-2 may not represent an ideal target for anti-angiogenenic tumor therapy.

In FGFR1-mediated intracellular signaling events, FGFR substrate 2 (FRS2) binds to the intracellular juxtamembrane domain of FGFR1, which associates with the two adaptor proteins Gab1 and Grb2, and tyrosine phosphatase Shp2 (48-50). The Grb2-Sos complex activates the Ras/MAPK pathway, which is downregulated by Sprouty (Spry) proteins in endothelial cells $(51,52)$. Phospholipase C-gamma directly binds to phosphorylated Y766 at the C-terminal tail of FGFR1, leading to activation of classical protein kinase C (53, 54). Shc, Shb, the Src family tyrosine kinases, signal transducer and activator of transcription (STAT), and p38 MAPK are also tyrosine phosphorylated and/or 
activated upon FGFR1 stimulation $(55,56)$.

Furthermore, Fes is activated by FGF-2 treatment in cultured endothelial cells via FGFR1, but not via FGFR2 (11). Activation of Fes depends on the culture conditions of endothelial cells. When murine brain capillary endothelial cells, or IBE cells (44), are cultured on the surface of a fibronectin-coated plastic substrate, c-Fes is activated by FGF-2 treatment; however, when IBE cells are cultured on three-dimensional collagen gels, c-Fes is only weakly activated independently of FGF-2 treatment (unpublished observations). Expression of kinase-inactive c-Fes exerts a dominant-negative effect on endogenous c-Fes, plausibly through oligomerization followed by the inhibition of autophosphorylation in trans (57). It has been reported that c-Fes is involved in FGF-2-directed chemotaxis by endothelial cells. One of the signaling molecules downstream of C-Fes is C-Src, which is recruited within focal adhesions (FAs) (58). In cells of non-endothelial origin, Gab1 forms a complex with Grb2 and FRS2, which enables tyrosine phosphorylation of Gab1 (50). Phosphorylated Gab1 then binds to the p85 regulatory subunit of PI3-kinase to recruit PI3-kinase to the plasma membrane, which in turn activates the downstream signaling molecule, c-Akt. However, FGFR1 fails to activate PI3-kinase in a manner dependent on the association with tyrosine-phosphorylated proteins in endothelial cells (59). Accordingly, expression of kinase-inactive c-Fes does not affect FGFR1-mediated activation of PI3-kinase (57), which was mainly induced by activated Ras (59). The mechanisms by which FGFR1 activates c-Fes and c-Fes activates c-Src within FA remain unclear.

\subsection{Angiopoietins}

Ang1-4 constitute a family of cytokines that are ligands for the receptor tyrosine kinase Tie 2 (60). Although the functions of Ang3 and 4 are less understood, Ang1 and 2 have been extensively studied. Ang1 was identified as an agonist of Tie 2 kinase. While targeted deletion of the tie2 or angiopoietin1 genes in mice proved to be fetal lethal with defects in late-stage vascular development $(61,62)$, overexpression of Ang2 in mice also disrupted vascular development, indicating that Ang2 antagonizes Ang1 (63). However, it was found later that Ang2 can activate Tie 2 in a context-dependent manner and is a key molecule for lymphatic vessel development (64-66). Ang1 and 2 require 
homo-oligomerization with a distinct number of each molecule to activate Tie 2 (67). In a confluent culture of endothelial cells, Ang1 induces Tie 2 dimerization across the cell-cell junction, resulting in an anti-apoptotic effect and the inhibition of permeability, whereas extracellular matrix-bound Ang1 dimerizes Tie 2 to promote migration $(68,69)$. These data may support the context-dependent activation of Tie 2 during angiogenesis. In addition, three closely located tyrosine residues (Y1101, Y1107, and Y1112) are autophosphorylated, and a number of intracellular signaling molecules, such as Grb2, 7, and 14, Dok-R, ShcA, Shp2, and the p85 subunit of class I PI3-kinase bind to these phosphorylated tyrosine residues (70). How these tyrosine residues target binding of particular signaling molecules remains unknown.

In some preclinical tumor models, both Ang1 and 2 are involved in angiogenesis and subsequent tumor progression (71-76). Selective inhibition of Ang2 activity suppresses angiogenesis and tumor growth in mice (77). Inhibition of Ang2 is additive with VEGF-A blockade (78) and has proved effective in a clinical trial with advanced cancers (79). Thus, Tie 2 signaling would be a potential target for anti-angiogenic therapy.

Downstream of Tie 2, Fes is autophosphorylated upon ligand stimulation $(65,80)$. In endothelial cells, which express endogenous c-Fes, activation of Tie 2 results in PI3-kinase activation, which is associated with c-Fes but not with Tie 2 (80). Expression of kinase-inactive c-Fes reduces Tie 2-mediated PI3-kinase activation, suggesting that c-Fes is required for Tie 2-mediated activation of PI3-kinase in endothelial cells $(65,80)$. Furthermore, it has been proposed that Tie 2-activated PI3-kinase is involved in survival and migration (81). Thus, blocking c-Fes may be a favorable strategy for anti-angiogenic therapy targeting Tie 2.

\subsection{Stromal cell-derived factor-1alpha}

SDF-1alpha (also known as CXCL12) is one of the CXC chemokines, which share a consensus Glu-Leu-Arg motif preceding the first cysteine residue. Its cognate receptor is CXCR4 (also known as CD184), which is a seven-transmembrane domain heterotrimeric G-protein-coupled receptor that is ubiquitously expressed, including in endothelial cells and hematopoietic cells. Its expression is regulated by the proangiogenic factors FGF-2 and VEGF-A (82), 
and targeted disruption of the cxcr4 gene results in defective vasculogenesis in intestine (83). SDF-1alpha/CXCR4 signaling is essential for mobilization of endothelial progenitor cells from the bone marrow and recruitment to the angiogenic niche (84). SDF-1alpha recruits smooth muscle progenitor cells from bone marrow to repair vascular wall injury (85) and is also implicated in the proliferation of some tumor cells (86).

SDF-1alpha binding to CXCR4 rapidly induces tyrosine phosphorylation and subsequent activation of JAK family kinases, which are involved in the induction of calcium influx and chemotaxis (87). However, diverse results have also been reported in HEK293 cells (88). Binding of SDF-1alpha to CXCR4 results in its activity as a guanine nucleotide exchange factor for the G-alpha subunit, which in turn enables dissociation with the G-beta-gamma subunits. PI3-kinase is activated through the G-beta-gamma subunits (89), and increased inositol triphosphate enhances c-Src activity and subsequent activation of Ras/MAPK pathway (90).

In endothelial cells, c-Fes also contributes to SDF-1alpha-mediated PI3-kinase activation (91). Expression of dominant negative c-Fes in endothelial cells abrogates tyrosine-phosphorylated protein-bound PI3-kinase activation. During tube formation of endothelial cells, VE-cadherin-dependent tight cell-cell contact is induced through SDF-1alpha-mediated PI3-kinase activation (91). Among three isoforms of the p110 catalytic subunit of class IA PI3-kinases (alpha , beta, and delta), Graupera et al. found that only p110alpha was indispensible for vascular development (92). p110alpha is preferentially activated via receptor tyrosine kinases. Downstream of G-protein-coupled receptors, such as CXCR4, p110beta is strongly activated in endothelial cells. Whether inhibition of the p110beta subunit of PI3-kinase in endothelial cells blocks tumor angiogenesis, or whether the c-Fes-activated catalytic subunit of PI3-kinase is p110beta remains to be determined.

\subsection{IL-8}

IL-8 (also known as CXCL8) is a proinflammatory chemokine with proangiogenic activity. Its receptors are CXCR1 and 2. IL-8 binding to the receptors promotes conformational changes and induces coupling of the receptor $\mathrm{C}$-terminal tail to heterotrimeric $\mathrm{G}$ proteins, similar to the behavior of 
CXCR4. However, CXCR1 and 2 exhibit distinct ligand-binding specificities and plausibly bind to different sets of G-alpha-beta-gamma combinations. Thus, intracellular signaling pathways are more complicated in IL-8-treated cells than in SDF-1alpha-treated cells. IL-8 stimulates proliferation, survival, and migration of tumor cells in an autocrine fashion, promotes neutrophil infiltration into tumor tissue, induces angiogenesis, and stimulates cytokine secretion by macrophages (93).

IL-8 activates PI3-kinase, the Ras/MAPK pathway, and phospholipase C (93). Interestingly, IL-8 transactivates several receptor tyrosine kinases, such as epidermal growth factor receptor and VEGFR-2 (94-97). Therefore, targeting IL-8 signaling may affect other proangiogenic factor-driven responses.

IL-8-activated signaling pathways in endothelial cells remain largely elusive. The role of c-Fes in IL-8-treated endothelial cells also is unknown. We examined IL-8-directed chemotaxis of porcine aortic endothelial (PAE) cells expressing either wild-type c-Fes (WT-10 cells) or kinase-inactive c-Fes (KE-12 cells). Chemotaxis toward FGF-2 by WT-10 cells remained intact, whereas that of KE-12 cells was impaired (98), plausibly through a dominant-negative effect of exogenously expressed kinase-inactive c-Fes on the endogenously expressed protein that has been observed in mouse brain capillary endothelial cells (57). As shown in Figure 1A, parental PAE cells dose dependently migrated toward IL-8, which was blocked by the PI3-kinase inhibitor LY294002, but not by the MEK inhibitor PD98059, suggesting that IL-8-directed chemotaxis is PI3-kinase dependent. WT-10 cells migrated efficiently toward IL-8, whereas IL-8-directed chemotaxis was impaired in KE-12 cells (Fig. 1B). These results indicate that IL-8-directed chemotaxis of PAE cells is c-Fes activity dependent, and downstream of c-Fes, the PI3-kinase/c-Akt pathway may be involved. As expected, IL-8 treatment increased phosphorylation of c-Akt Ser473 in WT-10 cells, but not in KE-12 cells (Fig. 1C). Taken together, these data suggest that IL-8 may participate in in vivo angiogenesis through the c-Fes/PI3-kinase/c-Akt pathway.

\subsection{VEGF}

VEGF-A is a key player for vascular development and pathophysiological angiogenesis (12). VEGF-A activates c-Fes via VEGFR-2 in cultured endothelial 
cells (99). Knock-out of a single vegf-a allele or of both vegfr-2 alleles in mice results in embryonic lethality because of the lack of normal vascular development (100-102). Expression of a membrane-targeted form of c-Fes mutant (via addition of an $\mathrm{N}$-terminal myristoylation signal sequence) in VEGFR-2-null embryonic stem cells partially rescues their vasculogenic and angiogenic properties in developing embryos, indicating that activated c-Fes may function downstream of VEGFR-2 during development (103). Endothelial cells expressing this membrane-targeted form of c-Fes mutant were hypersensitive to PDGF and VEGF-A(104), suggesting that some signals for c-Fes activation may emanate from membrane-bound angiogenic receptors.

To gain insight into the role of c-Fes in VEGF-A signaling, we examined the signal transduction pathways stimulated by VEGF-A in VEGFR-2-expressing PAE cells (99). The cells migrated and formed a capillary tube-like structure in response to VEGF-A treatment, and the PI3-kinase inhibitor LY294002 blocked these processes. We then focused on VEGF-A-induced PI3-kinase activation in these cells. Activated PI3-kinase associated with not only activated c-Fes but also tyrosine-phosphorylated c-Src, VEGFR-2, and insulin receptor substrate-I (99). Expression of kinase-inactive c-Fes abrogated the association of activated PI3-kinase with c-Fes, but other signaling molecules efficiently associated with VEGF-A-activated PI3-kinase. Accordingly, expression of kinase-inactive c-Fes failed to show a dominant-negative effect on VEGF-A-mediated migration and formation of a capillary tube-like structure. Thus, inhibition of c-Fes might not be an effective anti-angiogenic strategy for VEGF-A-driven tumor angiogenesis.

Interestingly, overexpression of wild-type c-Fes, but not of kinase-inactive c-Fes, results in a proangiogenic factor-independent, morphologically differentiated phenotype in endothelial cells when cultured in three-dimensional extracellular matrix proteins [IBE cells in collagen gels (57) or on Matrigel, and PAE cells on Matrigel (99)]. A similar phenotype has been observed in yolk-sac-derived endothelial cells from transgenic mice expressing artificially myristoylated, membrane-associated c-Fes (105). These results suggest that morphological differentiation may be quite sensitive to the elevation of basal kinase activity of c-Fes. We have observed that downregulation of c-Fes by siRNA in human umbilical vein endothelial cells results in decreased 
VEGF-A-dependent migration and morphological differentiation (106). Also, we noted that downregulation of c-Fes in IBE cells by siRNA attenuated cellular spreading on a fibronectin-coated surface with decreased FA formation, which was not seen in cells expressing kinase-inactive c-Fes (58). Morphological differentiation requires proper formation of FA (107). c-Fes may regulate FA formation by a kinase activity-independent scaffold function to recruit particular proteins to form a c-Fes-containing signaling complex. 


\section{FUTURE DIRECTIONS FOR THERAPUETIC APPLICATIONS}

As discussed above, c-Fes may be a common and significant signaling molecule in several proangiogenic factor-driven angiogenic pathways. Mice targeted with either null or kinase-inactivating mutations in c-Fes develop normally with no apparent defects in vasculogenesis or angiogenesis (108-110). This establishes that c-Fes is not required for developmental vasculogenesis or angiogenesis. c-Fes-deficient female mice are also capable of supporting multiple pregnancies; so adult physiological angiogenesis does not seem to require c-Fes. On the other hand, transgenic mice expressing activated c-Fes allele displayed developmental hypervascularity, which argues that c-Fes can participate in angiogenic signaling (111). The possibility that this hypervascular phenotype is unrelated to normal c-Fes function is still an outstanding question, but the high level of c-Fes expression observed in vascular endothelial cells would seem to argue that c-Fes does play a role in these cells. Thus, it is plausible that transient inhibition of c-Fes expression or activity by siRNA knock-down, expression of dominant-negative mutants, or treatment with selective small molecular weight synthetic kinase inhibitors, may inhibit angiogenesis to improve or stabilize disease progression. In particular, orally available c-Fes kinase inhibitors would be favorable for patients with advanced cancers because of the ease of administration and potential for low cost.

Should a putative c-Fes inhibitor be used as a first-line therapy? In particular, VEGF-A is a key player in almost all known pathological angiogenic processes, and even when c-Fes activity is specifically inhibited, VEGF-A-promoted PI3-kinase/Akt activation will likely be managed by other signaling molecules (99). Rather, c-Fes inhibition should be considered as a second-line therapy when tumors become refractory to VEGF-targeting therapies, or in combination with existing VEGF-directed drugs.

In recent years, a number of important lines of evidence have shown that bone marrow-derived monocytic cells are recruited into tumor tissue and play pivotal roles for angiogenesis and vasculogenesis (Fig. 2). Tumor cells secrete VEGF-A to recruit circulating endothelial progenitor cells, which are incorporated into the tumor vasculature (112). SDF-1alpha secreted by tumor-associated fibroblasts also contributes to the recruitment of progenitor cells (113), and tumor cells also can secrete SDF-1alpha. Tie 2-expressing monocytes are a specific 
subpopulation of bone marrow-derived proangiogenic cells, recruited by tumor cell-secreted Ang2 (114) and promoting angiogenesis by producing FGF-2 (115). Tumor-associated macrophages (TAM), kept in the M2 phenotype (protumoral state) by tumor-produced macrophage-colony stimulating factor, IL-10, and transforming growth factor-beta (116), secrete a number of proangiogenic cytokines, such as VEGF-A, FGF-2, HGF, PDGF, angiopoietin, IL-8, and epidermal growth factor (117). $\mathrm{CD} 11 \mathrm{~b}^{+} \mathrm{Gr} 1^{+}$cells are granulocytic and monocytic lineages and accumulate in tumor tissues refractory to anti-VEGF therapy (36). They produce Bv8 to induce angiogenesis in response to tumor cell-secreted granulocyte-colony stimulating factor (118). Most of these monocytes/macrophages probably express c-Fes; however, the roles of c-Fes in these cells are totally unknown. To explore the function of c-Fes in monocytic lineages recruited in tumor tissue, development of a c-Fes-specific synthetic inhibitor would be a necessity. 


\section{ACKNOWLEDGMENT}

This work was supported in part by Grants-in-Aid for Scientific Research from the Japan Society of the Promotion of Science to S.K. and Y.M. and the Research Award from the Nagasaki Prefectural Medical Association to S.K. 


\section{REFERENCES}

1. J. Folkman: Angiogenesis in cancer, vascular, rheumatoid and other disease. Nat Med 1, 27-31 (1995)

2. P. Carmeliet and R.K. Jain: Angiogenesis in cancer and other diseases. Nature 407, 249-257 (2000)

3. L. Beck Jr and P.A. D'Amore: Vascular development: cellular and molecular regulation. FASEB J 11, 365-373 (1997)

4. S. Klein, M. Roghani and D.B. Rifkin: Fibroblast growth factors as angiogenesis factors: new insights into their mechanism of action. EXS 79, 159-192 (1997)

5. N. Ferrara: Molecular and biological properties of vascular endothelial growth factor. $\mathrm{J} \mathrm{Mol}$ Med 77, 527-543 (1999)

6. E.M. Rosen, K. Lamszus, J. Laterra, P.J. Polverini, J.S. Rubin and I.D. Goldberg: HGF/SF in angiogenesis. Ciba Found Symp 212, 215-226 (1997)

7. W.S. Shim, I.A. Ho and P.E. Wong: Angiopoietin: a TIE(d) balance in tumor angiogenesis. Mol Cancer Res 5, 655-665 (2007)

8. B. Vanhaesebroeck, J. Guillermet-Guibert, M. Graupera and B. Bilanges: The emerging mechanisms of isoform-specific PI3K signalling. Nat Rev Mol Cell Biol 11, 329-341 (2010)

9. K.B. Reddy, S.M. Nabha and N. Atanaskova: Role of MAP kinase in tumor progression and invasion. Cancer Metastasis Rev 22, 395-403 (2003)

10. J.M. Summy and G.E. Gallick: Src family kinases in tumor progression and metastasis. Cancer Metastasis Rev 22, 337-358 (2003)

11. S. Kanda, Y. Miyata, H. Kanetake and T.E. Smithgall: Non-receptor protein-tyrosine kinases as molecular targets for antiangiogenic therapy (Review). Int J Mol Med 20, 113-121 (2007)

12. N. Ferrara, H.-P. Gerber and J. LeCouter: The biology of VEGF and its receptor. Nature Med 9, 669-676 (2003)

13. R.K. Jain: Molecular regulation of vessel maturation. Nat Med 9, 685-693 (2003)

14. M. Shibuya and L. Claesson-Welsh: Signal transduction by VEGF receptors in regulation of angiogenesis and lymphangiogenesis. Exp Cell Res 312, 549-560 (2006)

15. H. Gerhardt, M. Golding, M. Fruttiger, C. Ruhrberg, A. Lundkvist, A. Abramsson, M. Jeltsch, C. Mitchell, K. Alitalo, D. Shima and C. Betsholtz: VEGF guides angiogenic sprouting utilizing endothelial tip cell filopodia. J Cell Biol 161, 1163-1177 (2003)

16. M. Hellström, L.K. Phng, J.J. Hofmann, E. Wallgard, L. Coultas, P. Lindblom, J. Alva, A.K. Nilsson, L. Karlsson, N. Gaiano, K. Yoon, J. Rossant, M.L. Iruela-Arispe, M. Kalén, H. Gerhardt and C. Betsholtz: DII4 signalling through Notch1 regulates formation of tip cells during angiogenesis. Nature 445, 776-780 (2007)

17. L.S. Harrington, R.C. Sainson, C.K. Williams, J.M. Taylor, W. Shi, J.L. Li and A.L. Harris: Regulation of multiple angiogenic pathways by DIll 4 and Notch in human umbilical vein endothelial cells. Microvasc Res 75, 144-154 (2008)

18. S. Suchting, C. Freitas, F. le Noble, R. Benedito, C. Bréant, A. Duarte and A. Eichmann: The Notch ligand Delta-like 4 negatively regulates endothelial tip cell formation and vessel branching. Proc Natl Acad Sci U S A 104, 3225-3230 (2007)

19. R. Benedito, C. Roca, I. Sörensen, S. Adams, A. Gossler, M. Fruttiger and R.H. Adams: The notch ligands DII4 and Jagged1 have opposing effects on angiogenesis. Cell 137, 1124-1135 (2009)

20. A.F. Siekmann and N.D. Lawson: Notch signalling limits angiogenic cell behaviour in developing zebrafish arteries. Nature 445, 781-784 (2007)

21. T. Tammela, G. Zarkada, E. Wallgard, A. Murtomäki, S. Suchting, M. Wirzenius, M. Waltari, M. Hellström, T. Schomber, R. Peltonen, C. Freitas, A. Duarte, H. Isoniemi, P. Laakkonen, G. Christofori, S. Ylä-Herttuala, M. Shibuya, B. Pytowski, A. Eichmann, C. Betsholtz, K. Alitalo: Blocking VEGFR-3 suppresses angiogenic sprouting and vascular network formation. Nature 454, 656-660 (2008)

22. S. Sawamiphak, S. Seidel, CL. Essmann, G.A. Wilkinson, M.E. Pitulescu, T. Acker and A. Acker-Palmer: Ephrin-B2 regulates VEGFR2 function in developmental and tumour angiogenesis. Nature 2010: doi:10.1038/nature08995

23. Y. Wang, M. Nakayama, M.E. Pitulescu, T.S. Schmidt, M.L. Bochenek, A. Sakakibara, S. 
Adams, A. Davy, U. Deutsch, U. Lüthi, A. Barberis, L.E. Benjamin, T. Mäkinen, C.D. Nobes and R.H. Adams: Ephrin-B2 controls VEGF-induced angiogenesis and lymphangiogenesis. Nature 2010: doi:10.1038/nature09002

24. S.Y. Rabbany, B. Heissig, K. Hattori and S. Rafii: Molecular pathways regulating mobilization of marrow-derived stem cells for tissue revascularization. Trends Mol Med 9, 109-117 (2003)

25. J. Holash, P.C. Maisonpierre, D. Compton, P. Boland, C.R. Alexander, D. Zagzag, G.D. Yancopoulos and S.J. Wiegand: Vessel cooption, regression, and growth in tumors mediated by angiopoietins and VEGF. Science 284, 1994-1998 (1999)

26. R.S. Kerbel: A cancer therapy resistant to resistance. Nature 390, 335-336 (1997)

27. VEGF-targeted therapy: mechanisms of anti-tumour activity. Nature Rev Cancer 8, 579-591 (2008)

28. G. Bergers and D. Hanahan: Modes of resistance to anti-angiogenic therapy. Nature Rev Cancer 8, 592-603 (2008)

29. N. Ferrara: Pathways mediating VEGF-independent tumor angiogenesis. Cytokine Growth factor Rev 21, 21-26 (2010)

30. O. Casanovas, D.J. Hicklin, G. Bergers and D. Hanahan: Drug resistance by evasion of antiangiogenic targeting of VEGF signaling in late-stage pancreatic islet tumors. Cancer Cell 8 , 299-309 (2005)

31. J. Glade Bender, E.M. Cooney, J.J. Kandel and D.J. Yamashiro: Vascular remodeling and clinical resistance to antiangiogenic cancer therapy. Drug Resist Updat 7, 289-300 (2004)

32. L. Xu, D.G. Duda, E. di Tomaso, M. Ancukiewicz, D.C. Chung, G.Y. Lauwers, R. Samuel, P. Shellito, B.G. Czito, P.C. Lin, M. Poleski, R. Bentley, J.W. Clark, C.G. Willett and R.K. Jain: Direct evidence that bevacizumab, an anti-VEGF antibody, up-regulates SDF1alpha, CXCR4, CXCL6, and neuropilin 1 in tumors from patients with rectal cancer. Cancer Res 69, 7905-7910 (2009)

33. D. Huang, Y. Ding, M. Zhou, B.I. Rini, D. Petillo, C.N. Qian, R. Kahnoski, P.A. Futreal, K.A. Furge and B.T. The: Interleukin-8 mediates resistance to antiangiogenic agent sunitinib in renal cell carcinoma. Cancer Res 70, 1063-1071 (2010)

34. Y. Crawford, I. Kasman, L. Yu, C. Zhong, X. Wu, Z. Modrusan, J. Kaminker and N. Ferrara: PDGF-C mediates the angiogenic and tumorigenic properties of fibroblasts associated with tumors refractory to anti-VEGF treatment. Cancer Cell 15, 21-34 (2009)

35. G. Bergers, S. Song, N. Meyer-Morse, E. Bergsland and D. Hanahan: Benefits of targeting both pericytes and endothelial cells in the tumor vasculature with kinase inhibitors. $J$ Clin Invest 111, 1287-1295 (2003)

36. F. Shojaei, X. Wu, A.K. Malik, C. Zhong, M.E. Baldwin, S. Schanz, G. Fuh, H.P. Gerber and N. Ferrara: Tumor refractoriness to anti-VEGF treatment is mediated by $\mathrm{CD} 11 \mathrm{~b}+\mathrm{Gr} 1+$ myeloid cells. Nat Biotechnol 25, 911-920 (2007)

37. J. Vandercappellen, J. Van Damme and S. Struyf: The role of CXC chemokines and their receptors in cancer. Cancer Lett 267, 226-244 (2008)

38. Y. Masuda, Y. Takatsu, Y. Terao, S. Kumano, Y. Ishibashi, M. Suenaga, M. Abe, S. Fukusumi, T. Watanabe, Y. Shintani, T. Yamada, S. Hinuma, N. Inatomi, T. Ohtaki, H. Onda and M. Fujino: Isolation and identification of EG-VEGF/prokineticins as cognate ligands for two orphan G-protein-coupled receptors. Biochem Biophys Res Commun 293, 396-402 (2002)

39. J. Haigh, J. McVeigh and P. Greer: The fps/fes tyrosine kinase is expressed in myeloid, vascular endothelial, epithelial, and neuronal cells and is localized in the trans-golgi network. Cell Growth Differ 7, 931-944 (1996)

40. N. Itoh and D.M. Ornitz: Evolution of the Fgf and Fgfr gene families. Trends Genet 20, 563-569 (2004)

41. P. Klint and L. Claesson-Welsh: Signal transduction by fibroblast growth factor receptors. Front Biosci 4, D165-177 (1999)

42. M. Rusnati and M. Presta: Fibroblast growth factors/fibroblast growth factor receptors as targets for the development of anti-angiogenesis strategies. Curr Pharm Des 13, 2025-2044 (2007)

43. D.M. Ornitz, J. Xu, J.S. Colvin, D.G. McEwen, C.A. MacArthur, F. Coulier, G. Gao and M. Goldfarb: Receptor specificity of the fibroblast growth factor family. J Biol Chem 271, 
15292-15297 (1996)

44. S. Kanda, E. Landgren, M. Ljungström and L. Claesson-Welsh: Fibroblast growth factor receptor 1-induced differentiation of endothelial cell line established from tsA58 large T transgenic mice. Cell Growth Differ 7, 383-395 (1996)

45. T. Nakamura, Y. Mochizuki, H. Kanetake and S. Kanda: Signals via FGF receptor 2 regulate migration of endothelial cells. Biochem Biophys Res Commun 289, 801-806 (2001)

46. S. Ortega, M. Ittmann, S.H. Tsang, M. Ehrlich and C. Basilico: Neuronal defects and delayed wound healing in mice lacking fibroblast growth factor 2. Proc Natl Acad Sci U S A 95, 5672-5677 (1998)

47. A. Montero, Y. Okada, M. Tomita, M. Ito, H. Tsurukami, T. Nakamura, T. Doetschman, J.D. Coffin and M.M. Hurley: Disruption of the fibroblast growth factor-2 gene results in decreased bone mass and bone formation. J Clin Invest 105, 1085-1093 (2000)

48. H. Xu, K.W. Lee and M. Goldfarb: Novel recognition motif on fibroblast growth factor receptor mediates direct association and activation of SNT adapter proteins. J Biol Chem 273, 17987-17990 (1998)

49. Y.R. Hadari, H. Kouhara, I. Lax and J. Schlessinger: Binding of Shp2 tyrosine phosphatase to FRS2 is essential for fibroblast growth factor-induced PC12 cell differentiation. Mol Cell Biol 18, 3966-3973 (1998)

50. S.H. Ong, Y.R. Hadari, N. Gotoh, G.R. Guy, J. Schlessinger and I. Lax: Stimulation of phosphatidylinositol 3-kinase by fibroblast growth factor receptors is mediated by coordinated recruitment of multiple docking proteins. Proc Natl Acad Sci U S A 98, 6074-6079 (2001)

51. S.H. Lee, D.J. Schoss, L. Jarvis, M.A. Krasnow and J.L. Swain: Inhibition of angiogenesis by a mouse sprouty protein. J Biol Chem 276, 4128-4133 (2001)

52. M.A. Impagnatiello, S. Weitzer, G. Gannon, A. Compagni, M. Cotten annd G. Christofori: Mammalian sprouty-1 and -2 are membrane-anchored phosphoprotein inhibitors of growth factor signaling in endothelial cells. J Cell Biol 152, 1087-1098 (2001)

53. M. Mohammadi, C.A. Dionne, W. Li, N. Li, T. Spivak, A.M. Honegger, M. Jaye and J. Schlessinger: Point mutation in FGF receptor eliminates phosphatidylinositol hydrolysis without affecting mitogenesis. Nature 358, 681-684 (1992)

54. K.G. Peters, J. Marie, E. Wilson, H.E. Ives, J. Escobedo, M. Del Rosario, D. Mirda and L.T. Williams: Point mutation of an FGF receptor abolishes phosphatidylinositol turnover and $\mathrm{Ca} 2+$ flux but not mitogenesis. Nature 358, 678-681 (1992)

55. S. Javerzat, P. Auguste and A. Bikfalvi: The role of fibroblast growth factors in vascular development. Trends Mol Med 8, 483-489 (2002)

56. M.J. Cross and L. Claesson-Welsh: FGF and VEGF function in angiogenesis: signalling pathways, biological responses and therapeutic inhibition. Trends Pharmacol Sci 22, 201-207 (2001)

57. S. Kanda, E.C. Lerner, S. Tsuda, T. Shono, H. Kanetake and T.E. Smithgall: The nonreceptor protein-tyrosine kinase c-Fes is involved in fibroblast growth factor-2-induced chemotaxis of murine brain capillary endothelial cells. J Biol Chem 275, 10105-10111 (2000)

58. S. Kanda, Y. Miyata, H. Kanetake and T.E. Smithgall: Fibroblast growth factor-2 induces the activation of Src through Fes, which regulates focal adhesion disassembly. Exp Cell Res 312, 3015-3022 (2006)

59. Y. Mochizuki, S. Tsuda, H. Kanetake and S. Kanda: Negative regulation of urokinase-type plasminogen activator production through FGF-2-mediated activation of phosphoinositide 3-kinase. Oncogene 21, 7027-7033 (2002)

60. S. Davis, N.W. Gale, J.S. Rudge, S.J. Wiegand, J. Holash and G.D. Yancopoulos: Vascular-specific growth factors and blood vessel formation. Nature 407:242-248 (2000)

61. D.J. Dumont, G. Gradwohl, G.-H. Fong, M.C. Puri, M. Gertsenstein, A. Auerbach and M.L. Breitman: Dominant-negative and targeted null mutations in the endothelial receptor tyrosine kinase, tek, reveal a critical role in vasculogenesis of the embryo. Genes Dev 8, 1897-1909 (1994)

62. C. Suri, P.F. Jones, S. Patan, S. Bartunkova, P.C. Maisonpierre, S. Davis, T.N. Sato and G.D. Yancopoulos: Requisite role of angiopoietin-1, a ligand for the TIE2 receptor, during embryonic angiogenesis. Cell 87, 1171-1180 (1996)

63. P.C. Maisonpierre, C. Suri, P.F. Jones, S. Bartunkova, S.J. Wiegand, C. Radziejewski, D. 
Compton, J. McClain, T.H. Aldrich, N. Papadopoulos, T.J. Daly, S. Davis, T.N. Sato and G.D. Yancopoulos: Angiopoietin-2, a natural antagonist for Tie2 that disrupts in vivo angiogenesis. Science 277, 55-60 (1997)

64. I. Kim, J.-H. Kim, S.-O. Moon, H.J. Kwak, N.-G. Kim and G.Y. Koh: Angiopoietin-2 at high concentration can enhance endothelial cell survival through the phosphatidylinositol 3'-kinase/Akt signal transduction pathway. Oncogene 19, 4549-4552 (2000)

65. Y. Mochizuki, T. Nakamura, H. Kanetake and S. Kanda: Angiopoietin 2 stimulates migration and tube-like structure formation of murine brain capillary endothelial cells through c-Fes and c-Fyn. J Cell Sci 115, 175-183 (2002)

66. N.W. Gale, G. Thurston, S.F. Hackett, R. Renard, Q. Wang, J. McClain, C. Martin, C. Witte, M.H. Witte, D. Jackson, C. Suri, P.A. Campochiaro, S.J. Wiegand and G.D. Yancopoulos: Angiopoietin-2 is required for postnatal angiogenesis and lymphatic patterning, and only the latter role is rescued by Angiopoietin-1. Dev Cell 3, 411-423 (2002)

67. S. Davis, N. Papadopoulos, T.H. Aldrich, P.C. Maisonpierre, T. Huang, L. Kovac, A. Xu, R. Leidich, E. Radziejewska, A. Rafique, J. Goldberg, V. Jain, K. Bailey, M. Karow, J. Fandl, S.J. Samuelsson, E. loffe, J.S. Rudge, T.J. Daly, C. Radziejewski and G.D. Yancopoulos: Angiopoietins have distinct modular domains essential for receptor binding, dimerization and superclustering. Nat Struct Biol 10, 38-44 (2003)

68. S. Fukuhara, K. Sako, T. Minami, K. Noda, H.Z. Kim, T. Kodama, M. Shibuya, N. Takakura, G.Y. Koh and N. Mochizuki: Differential function of Tie2 at cell-cell contacts and cell-substratum contacts regulated by angiopoietin-1. Nat Cell Biol 10, 513-526 (2008)

69. P. Saharinen, L. Eklund, J. Miettinen, R. Wirkkala, A. Anisimov, M. Winderlich, A. Nottebaum, D. Vestweber, U. Deutsch, G.Y. Koh, B.R. Olsen and K. Alitalo: Angiopoietins assemble distinct Tie2 signalling complexes in endothelial cell-cell and cell-matrix contacts. Nat Cell Biol 10, 527-537 (2008)

70. K.G. Peters, C.D. Kontos, P.C. Lin, A.L. Wong, P. Rao, L. Huang, M.W. Dewhirst and S. Sankar: Functional significance of Tie2 signaling in the adult vasculature. Recent Prog Horm Res 59, 51-71 (2004)

71. T. Etoh, H. Inoue, S. Tanaka, G.F. Barnard, S. Kitano and M. Mori: Angiopoietin-2 is related to tumor angiogenesis in gastric carcinoma: possible in vivo regulation via induction of proteases. Cancer Res 61, 2145-2153 (2001)

72. W.S. Shim, M. Teh, A. Bapna, I. Kim, G.Y. Koh, P.O. Mack and R. Ge: Angiopoietin 1 promotes tumor angiogenesis and tumor vessel plasticity of human cervical cancer in mice. Exp Cell Res 279, 299-309 (2002)

73. T. Nakayama, L. Yao and G. Tosato: Mast cell-derived angiopoietin-1 plays a critical role in the growth of plasma cell tumors. J Clin Invest 114, 1317-1325 (2004)

74. M.R. Machein, A. Knedla, R. Knoth, S. Wagner, E. Neuschl and K.H. Plate: Angiopoietin-1 promotes tumor angiogenesis in a rat glioma model. Am J Pathol 165, 1557-1570 (2004)

75. R. Maffei, S. Martinelli, I. Castelli, R. Santachiara, P. Zucchini, M. Fontana, S. Fiorcari, G. Bonacorsi, F. Ilariucci, G. Torelli and R. Marasca: Increased angiogenesis induced by chronic lymphocytic leukemia B cells is mediated by leukemia-derived Ang2 and VEGF. Leuk Res 34, 312-321 (2010)

76. K.M. Detjen, S. Rieke, A. Deters, P. Schulz, A. Rexin, S. Vollmer, P. Hauff, B. Wiedenmann, M. Pavel and A. Scholz: Angiopoietin-2 promotes disease progression of neuroendocrine tumors. Clin Cancer Res 16, 420-429 (2010)

77. J. Oliner, H. Min, J. Leal, D. Yu, S. Rao, E. You, X. Tang, H. Kim, S. Meyer, S.J. Han, N. Hawkins, R. Rosenfeld, E. Davy, K. Graham, F. Jacobsen, S. Stevenson, J. Ho, Q. Chen, T. Hartmann, M. Michaels, M. Kelley, L. Li, K. Sitney, F. Martin, J.R. Sun, N. Zhang, J. Lu, J. Estrada, R. Kumar, A. Coxon, S. Kaufman, J. Pretorius, S. Scully, R. Cattley, M. Payton, S. Coats, L. Nguyen, B. Desilva, A. Ndifor, I. Hayward, R. Radinsky, T. Boone and R. Kendall: Suppression of angiogenesis and tumor growth by selective inhibition of angiopoietin-2. Cancer Cell 6, 507-516 (2004)

78. H. Hashizume, B.L. Falcón, T. Kuroda, P. Baluk, A. Coxon, D. Yu, J.V. Bready, J.D. Oliner and D.M. McDonald: Complementary actions of inhibitors of angiopoietin-2 and VEGF on tumor angiogenesis and growth. Cancer Res 70, 2213-2223 (2010)

79. A.C. Mita, C.H. Takimoto, M. Mita, A. Tolcher, K. Sankhala, J. Sarantopoulos, M. Valdivieso, 
L. Wood, E. Rasmussen, Y.N. Sun, Z.D. Zhong, M.B. Bass, N. Le and P. Lorusso: Phase 1 Study of AMG 386, a Selective Angiopoietin 1/2-Neutralizing Peptibody, in Combination with Chemotherapy in Adults with Advanced Solid Tumors. Clin Cancer Res 16, 3044-3056 (2010)

80. S. Kanda, Y. Miyata, Y. Mochizuki, T. Matsuyama and H. Kanetake: Angiopoietin 1 is mitogenic for cultured endothelial cells. Cancer Res 65, 6820-6827 (2005)

81. N. Jones and D.J. Dumont: Tek/Tie2 signaling: new and old partners. Cancer Metastasis Rev 19, 13-17 (2000)

82. R. Salcedo, K. Wasserman, H.A. Young, M.C. Grimm, O.M. Howard, M.R. Anver, H.K. Kleinman, W.J. Murphy and J.J. Oppenheim: Vascular endothelial growth factor and basic fibroblast growth factor induce expression of CXCR4 on human endothelial cells: In vivo neovascularization induced by stromal-derived factor-1alpha. Am J Pathol 154, 1125-1135 (1999)

83. K. Tachibana, S. Hirota, H. lizasa, H. Yoshida, K. Kawabata, Y. Kataoka, Y. Kitamura, K. Matsushima, N. Yoshida, S. Nishikawa, T. Kishimoto and T. Nagasawa: The chemokine receptor CXCR4 is essential for vascularization of the gastrointestinal tract. Nature 393, 591-594 (1998)

84. I. Petit, D. Jin and S. Rafii: The SDF-1-CXCR4 signaling pathway: a molecular hub modulating neo-angiogenesis. Trend Immunol 28. 299-307 (2007)

85. A. Schober, S. Knarren, M. Lietz, E.A. Lin and C. Weber: Crucial role of stromal cell-derived factor-1alpha in neointima formation after vascular injury in apolipoprotein E-deficient mice. Circulation 108, 2491-2497 (2003)

86. J.B. Rubin: Chemokine signaling in cancer: one hump or two? Sem Cancer Biol 19, 116-122 (2009)

87. A.J. Vila-Colo, J.M. Rodriguez-Frade, A.M. De Ana, M.C. Moreno-Ortiz, C. Martinez-A and M. Mellado: The chemokine SDF-1 triggers CXCR4 receptor dimerization and activates the JAK/STAT pathway. FASEB J 13, 1699-1710 (1999)

88. M. Moriguchi, B.D. Hissong, M. Gadina, K. Yamaoka, H.L. Tiffany, P.M. Murphy, F. Candotti and J.J. O'Shea: CXCL12 signaling is independent of Jak2 and Jak3. J Biol Chem 280, 17408-17414 (2005)

89. L. Stephens, A. Smrcka, F.T. Cooke, T.R. Jackson, P.C. Sternweis and P.T. Hawkins: A novel phosphoinositide 3 kinase activity in myeloid-derived cells is activated by $\mathrm{G}$ protein beta gamma subunits. Cell 77, 83-93 (1994)

90. T. van Biesen, B.E. Hawes, D.K. Luttrell, K.M. Krueger, K. Touhara, E. Porfiri, M. Sakaue, L.M. Luttrell and R.J. Lefkowitz: Receptor-tyrosine-kinase- and G beta gamma-mediated MAP kinase activation by a common signalling pathway. Nature 376, 781-784 (1995)

91. S. Kanda, Y. Mochizuki and H. Kanetake: Stromal cell-derived factor-1alpha induces tube-like structure formation of endothelial cells through phosphoinositide 3-kinase. J Biol Chem 278, 257-262 (2003)

92. M. Graupera, J. Guillermet-Guibert, L.C. Foukas, L.K. Phng, R.J. Cain, A. Salpekar, W. Pearce, S. Meek, J. Millan, P.R. Cutillas, A.J. Smith, A.J. Ridley, C. Ruhrberg, H. Gerhardt and B. Vanhaesebroeck: Angiogenesis selectively requires the p110alpha isoform of PI3K to control endothelial cell migration. Nature 453, 662-666 (2008)

93. D.J. Waugh and C. Wilson: The interleukin-8 pathway in cancer. Clin Cancer Res 14, 6735-6741 (2008)

94. G. Venkatakrishnan, R. Salgia and J.E. Groopman: Chemokine receptors CXCR-1/2 activate mitogen-activated protein kinase via the epidermal growth factor receptor in ovarian cancer cells. J Biol Chem 275, 6868-6875 (2000)

95. I.U. Schraufstatter, K. Trieu, M. Zhao, D.M. Rose, R.A. Terkeltaub and M. Burger: IL-8-mediated cell migration in endothelial cells depends on cathepsin B activity and transactivation of the epidermal growth factor receptor. J Immunol 171, 6714-6722 (2003)

96. F. Luppi, A.M. Longo, W.I. de Boer, K.F. Rabe and P.S. Hiemstra: Interleukin-8 stimulates cell proliferation in non-small cell lung cancer through epidermal growth factor receptor transactivation. Lung Cancer 56, 25-33 (2007)

97. M.L. Petreaca, M. Yao, Y. Lui, K. Defea and M. Martins-Green: Transactivation of vascular endothelial growth factor receptor-2 by interleukin-8 (IL-8/CXCL8) is required for IL-8/CXCL8-induced endothelial permeability. Mol Biol Cell 18, 5014-5023 (2007) 
98. S. Kanda, A. Naba and Y. Miyata: Inhibition of endothelial cell chemotaxis toward FGF-2 by gefitinib associates with downregulation of Fes activity. Int J Oncol 35, 1305-1312 (2009)

99. S. Kanda, Y. Mochizuki, Y. Miyata and H. Kanetake: The role of c-Fes in vascular endothelial growth factor-A-mediated signaling by endothelial cells. Biochem Biophys Res Commun 306, 1056-1063 (2003)

100. N. Ferrara, K. Carver Moore, H. Chen, M. Dowd, L. Lu, K.S. O'Shea, L. Powell Braxton, K.J. Hillan and M.W. Moore: Heterozygous embryonic lethality induced by targeted inactivation of the VEGF gene. Nature 380, 439-442 (1996)

101. P. Carmeliet, V. Ferreira, G. Breier, S. Pollefeyt, L. Kieckens, M. Gertsenstein, M. Fahrig, A. Vandenhoeck, H. Kendraprasad, C. Eberhardt, C. Declercq, J. Pawling, L. Moons, D. Collen, W. Risau and A. Nagy: Abnormal blood vessel development and lethality in embryos lacking a single vascular endothelial growth factor allele. Nature 380, 435-439 (1996)

102. F. Shalaby, J. Rossant, T.P. Yamaguchi, M. Gertsenstein, X.F. Wu, M.L. Breitman and A.C. Schuh: Failure of blood-island formation and vasculogenesis in Flk-1-deficient mice. Nature 376, 62-66 (1995)

103. J.J. Haigh, M. Ema, K. Haigh, M. Gertsenstein, P. Greer, J. Rossant, A. Nagy and E.F. Wagner EF: Activated Fps/Fes partially rescues the in vivo developmental potential of Flk1-deficient vascular progenitor cells. Blood 103, 912-920 (2004)

104. W. Sangrar, J.D. Mewburn, S.G. Vincent, J.T. Fisher and P.A. Greer: Vascular defects in gain-of-function fps/fes transgenic mice correlate with PDGF- and VEGF-induced activation of mutant Fps/Fes kinase in endothelial cells. J Thromb Haemost 2, 820-832 (2004)

105. S.J. Wang, P. Greer and R. Auerbach: Isolation and propagation of yolk-sac-derived endothelial cells from a hypervascular transgenic mouse expressing gain-of-function fps/fes protooncogene. In Vitro Cell Dev Biol Anim 32, 292-299 (1996)

106. S. Kanda, H. Kanetake and Y. Miyata: Downregulation of Fes inhibits VEGF-A-induced chemotaxis and capillary-like morphogenesis by cultured endothelial cells. J Cell Mol Med 11, 495-501 (2007)

107. S. Kanda, Y. Miyata and H. Kanetake: Role of focal adhesion formation in migration and morphogenesis of endothelial cells. Cell Signal 16, 1273-1281 (2004)

108. Y. Senis, R. Zirngibl, J. McVeigh, A. Haman, T. Hoang T and P.A. Greer: Targeted disruption of the murine fps/fes proto-oncogene reveals that Fps/Fes kinase activity is dispensable for hematopoiesis. Mol Cell Biol 19, 7436-7446 (1999)

109. R. Hackenmiller, J. Kim, R.A. Feldman and M.C. Simon: Abnormal Stat activation, hematopoietic homeostasis, and innate immunity in c-fes-/- mice. Immunity 13, 397-407 (2000)

110. R.A. Zirngibl, Y. Senis and P.A. Greer: Enhanced endotoxin sensitivity in fps/fes-null mice with minimal defects in hematopoietic homeostasis. Mol Cell Biol 22, 2472-2486 (2002)

111. P. Greer, J. Haigh, G. Mbamalu, W. Khoo, A. Bernstein and T. Pawson: The Fps/Fes protein-tyrosine kinase promotes angiogenesis in transgenic mice. Mol Cell Biol 14, 6755-6763 (1994)

112. M. Grunewald, I. Avraham, Y. Dor, E. Bachar-Lustig, A. Itin, S. Jung, S. Chimenti, L. Landsman, R. Abramovitch and E. Keshet: VEGF-induced adult neovascularization: recruitment, retention, and role of accessory cells. Cell 124, 175-189 (2006)

113. A. Orimo, P.B. Gupta, D.C. Sgroi, F. Arenzana-Seisdedos, T. Delaunay, R. Naeem, V.J. Carey, A.L. Richardson and R.A. Weinberg: Stromal fibroblasts present in invasive human breast carcinomas promote tumor growth and angiogenesis through elevated SDF-1/CXCL12 secretion. Cell 121, 335-348 (2005)

114. C. Murdoch, S. Tazzyman, S. Webster and C.E. Lewis: Expression of Tie-2 by human monocytes and their responses to angiopoietin-2. J Immunol 178, 7405-7411 (2007)

115. M. De Palma, M.A. Venneri, R. Galli, L. Sergi Sergi, L.S. Politi, M. Sampaolesi and L. Naldini: Tie2 identifies a hematopoietic lineage of proangiogenic monocytes required for tumor vessel formation and a mesenchymal population of pericyte progenitors. Cancer Cell 8 , 211-226 (2005)

116. A. Mantovani, P. Allavena, A. Sica and F. Balkwill: Cancer-related inflammation. Nature 454, 436-444 (2008)

117. C. Lewis C and C. Murdoch: Macrophage responses to hypoxia: implications for tumor 
progression and anti-cancer therapies. Am J Pathol 167, 627-635 (2005)

118. F. Shojaei, X. Wu, C. Zhong, L. Yu, X.H. Liang, J. Yao, D. Blanchard, C. Bais, F.V. Peale, N. van Bruggen, C. Ho, J. Ross, M. Tan, R.A. Carano, Y.G. Meng and N. Ferrara: Bv8 regulates myeloid-cell-dependent tumour angiogenesis. Nature 450, 825-831 (2007) 


\section{FIGURE LEGENDS}

A

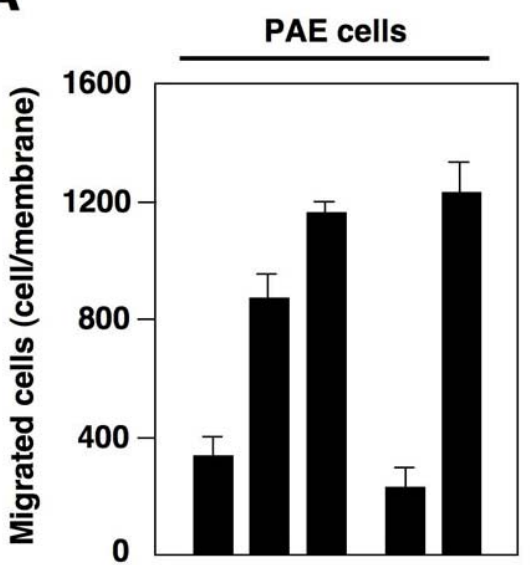

IL-8 (ng/ml) : 040200200200

$\operatorname{DMSO}(0.1 \%)$ : $\quad+\quad+\quad-\quad$ LY294002 (10 mM) : - - - - + PD98059 (50 mM) : $\quad$ - $\quad-\quad-\quad-\quad+$
B

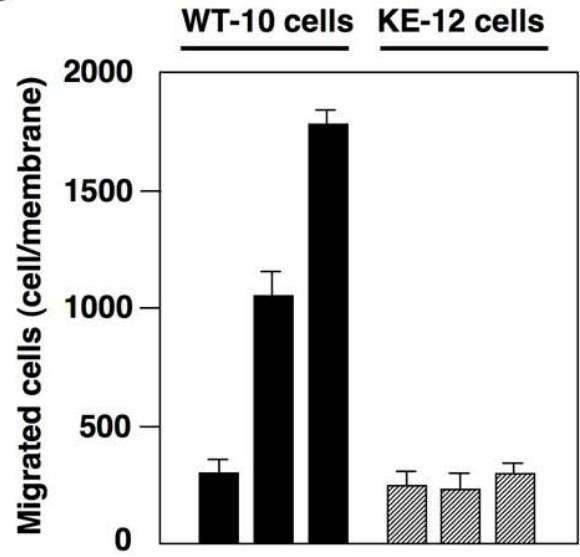

IL-8 (ng/ml) : $040200 \quad 0 \quad 40200$

\section{IP : Anti-Akt-1 \\ WT- 10 cells KE- 12 cells}

IL-8 : - + - +

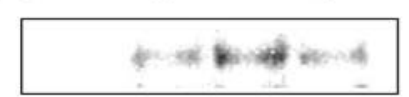

IB : Anti-phospho Akt-1 [S473]

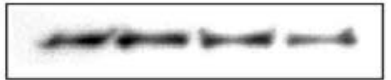

IB : Anti-Akt-1
Total cell lysate

WT- 10 cells KE-12 cells

IL-8

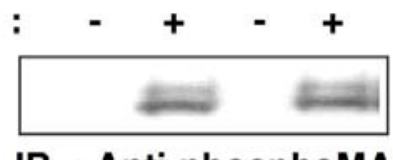

IB : Anti-phosphoMAPK [T202/Y204]

IB : Anti-ERK 1

Figure 1. IL-8-directed chemotaxis of endothelial cells is c-Fes/PI3-kinase dependent. (A) Culture media containing IL-8 (Peprotech, Inc.) at indicated concentrations with either DMSO $(0.1 \%)$, LY294002, or PD98059 were placed in the lower wells of Transwell culture plates, and PAE cells were seeded onto the upper surface of the Transwell insert precoated with type I collagen. Cells that migrated onto the lower surface of the Transwell inserts were counted. Reproducible results were obtained from three independent experiments. (B) PAE cells stably expressing wild-type Fes (WT-10 cells) and cells expressing kinase-inactive Fes (KE-12 cells) were seeded onto the upper surface of the Transwell insert, and culture media containing IL-8 at the indicated concentrations were placed in the lower wells of the Transwell plates. Reproducible results were obtained from two independent experiments. (C) 
WT-10 cells and KE-12 cells were serum starved overnight and then stimulated with IL-8 (at $200 \mathrm{ng} / \mathrm{ml}$ ) for $8 \mathrm{~min}$. c-Akt-1 was immunoprecipitated from $97 \%$ of total cell lysates with anti-c-Akt-1 monoclonal antibody (Santa Cruz Biotechnologies, Inc.), separated by SDS-PAGE, followed by transfer onto PVDF membranes. Phosphorylation of C-Akt-1 at $\$ 473$ was examined by immunoblotting with the use of anti-phospho-S473 antibody (Santa Cruz Biotechnologies, Inc.). The remaining $3 \%$ of cell lysates was mixed with SDS-sample buffer, and detection of activated MAPK with anti-phosphoMAPK antibody (Cell Signaling Technologies, Inc.) by immunoblotting was performed as described above. Reproducible results were obtained from two independent experiments. 


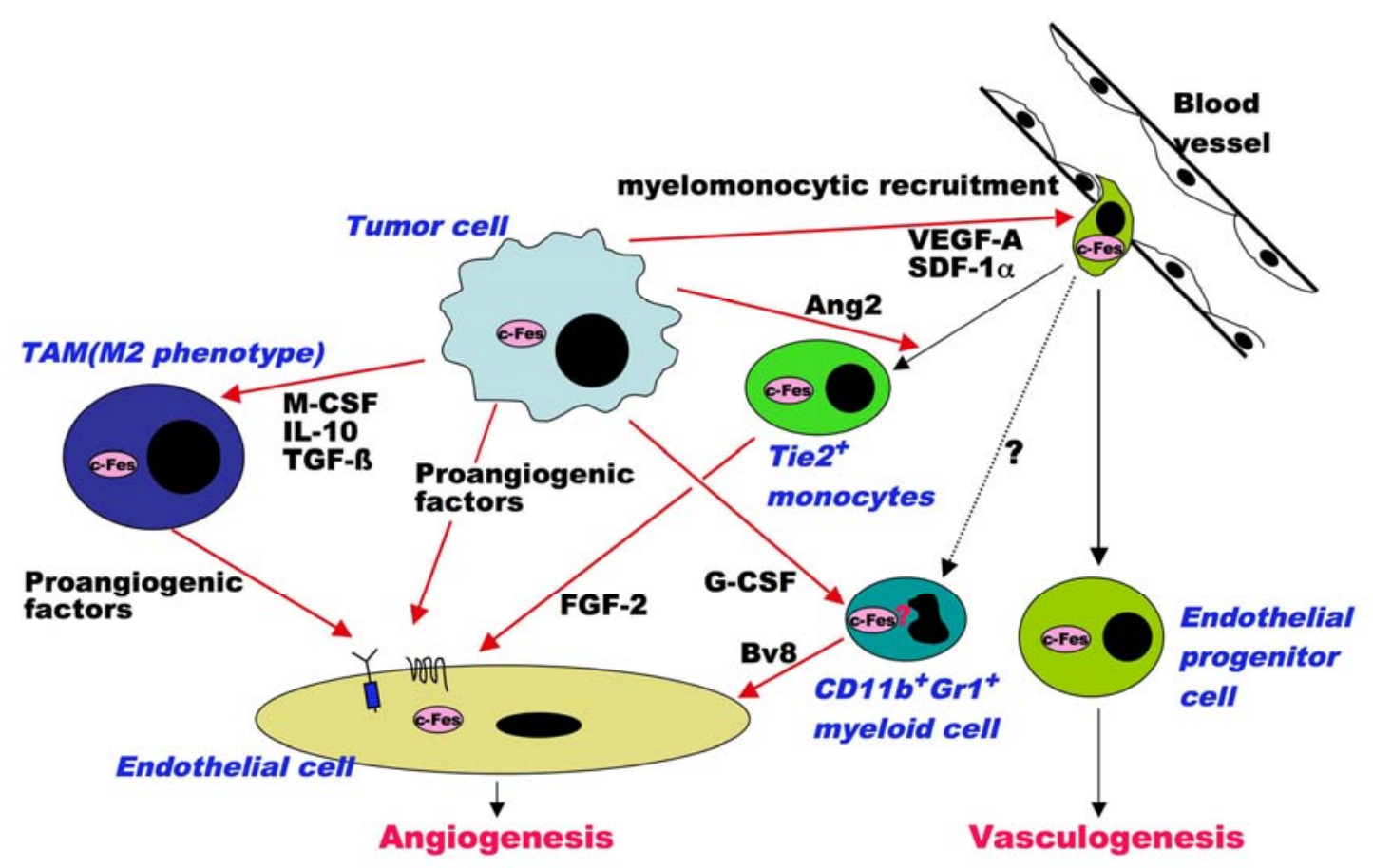

Figure 2. Proposed model of tumor angiogenesis and vasculogenesis, and plausible players in these cellular processes. Red arrows indicate the secreted cytokines acting on the target cells. Tumor cells themselves secrete a variety of proangiogenic factors to induce angiogenesis. Tumor cells secrete M-CSF and IL-10 and maintain TAM in the protumoral phase (M2 phenotype). Then, TAM secretes a number of proangiogenic cytokines, such as VEGF-A, FGF-2, angiopoietins, IL-8, SDF-1alpha, and TGF-beta to stimulate angiogenesis. VEGF-A and SDF-1alpha, which are secreted by tumor cells, recruit the monocyte lineage from bone marrow. From these, circulating endothelial progenitor cells are incorporated into tumor tissue to constitute a novel vessel (vasculogenesis). Tumor-secreted Ang2 stimulates Tie 2-expressing monocytes to produce FGF-2. In tumor tissues refractory to anti-VEGF therapies, $\mathrm{CD} 11 \mathrm{~b}^{+} \mathrm{Gr} 1^{+}$myeloid cells are accumulated and produce Bv8 in response to tumor cell-secreted G-CSF. Bv8 also stimulates angiogenesis. TAM, tumor-associated macrophages; Ang2, angiopoietin 2; Bv8, endocrine gland-derived VEGF homolog Bv8; FGF-2, fibroblast growth factor-2 (basic FGF); G-CSF, granulocyte colony stimulating factor; IL-10, interleukin-10; M-CSF, macrophage colony stimulating factor; SDF-1alpha, stromal cell-derived factor-1alpha (CXCL-12); TGF-beta, transforming growth factor-beta; VEGF, vascular endothelial growth factor. 